\title{
A Study of Word Formation of English Net Language
}

\author{
Li Liu \\ School of Foreign Languages, Zhengzhou University of Industrial Technology, Zhengzhou 451150, \\ China
}

625960018@qq.com

Keywords: English net language, word formation.

\begin{abstract}
The use of computers as a medium of communication has given birth to a new language phenomenon which is Net language. English net language is a product of the Internet and the times. A large number of English new vocabularies promote the diversified development of the English net language in a certain extent, at the same time this new phenomenon of language has attracted much attention. The English net language has gradually infiltrated into our daily life, so it is necessary to deeply analyze the formation and changes of English net language and forecasting the development of English net language and the influence on the social language in reality.
\end{abstract}

\section{Introduction}

With the popularization of computers and the Internet, the studies of English net language have entered a new stage. And there are many scholars having studied the English net language home and abroad.

English is the main language in computer network, and new words are mainly produced from English. That's why the recent research on the English net language is earlier in overseas, and later in China. While the special study on the English net language is relatively few. In 1977, a meeting called "Language and the Internet" was organized to talk about the net language in many aspects firstly. In 2001, a famous scholar David Crystal wrote a book named Language and the Internet. In this book, the definition of net language classification is firstly described in detail, and he thinks net words can enrich our language. Baron in his article Computer Mediated Communication as Force in Language Change pointed out the fact that the net language may replace the traditional written language and oral language and be regarded as a new way of language communication. Marketa Johnova makes a brief description of the word formation of English net language in The Language of Chat. He considers that the characteristic of English net language is the wide use of abbreviations and acronyms to accelerate communication and give English net language the features of colloquialism.

The studies on net language can be mainly divided into word formation and cognition in China. In word formation, Zhang Jing starts from the concept and classification of net language, then she studies the word formation of English net language and Chinese net language to analyze the differences. Through analysis, the writer draws such a conclusion that the ways of word formation of English net language are word interception, combination of words and letters, phrase acronyms, and sentence acronyms. With the development of Cognitive Linguistic Theory in recent years, it has been a new research approach to study words from a cognitive perspective. For example, Li Weiwei studies the forming reason mainly frommetaphor theory. While Sun Chongfei and Wang Henglan analyze the reason of word formation of net language based on the theory oficonicity.

\section{Definition of English Net Language}

Because of the Internet, a large number of English net words arise. Each of us could realize that the Internet has changed not only your lifestyles but also our language. In recent years, it is necessary for more and more people to use network in their daily lives, such as exchanging ideas, expressing personal emotion, doing network teaching, having network conferences, making Internet chat and so on. 
English net language is a new media language which is used in net communication. In the Netdictionary column of YAHOO website, the definition of the English net language is that standard Internet English are commonly used words in E-Mail, Discussion Group, Chat-rooms, WWW Pages and everywhere on the net, including those of technical terms, hackers slang and acronyms related with Internet. In other words, the English terminology of computer and network technology as well as terms which relate the phenomenon of network culture and Computer-mediated communication has composed the general concept of the English net language.

\section{Word Formation of English Net Language}

\subsection{Compound New Words}

Compound word formation is the most common method in English net vocabulary. In English network language, most of the emerging vocabularies are constructed in such a way.

A compound word is the combination of one or more letters from two or more words in order to form a English new word with a new meaning. The words are easy to be accepted and generalized. There are some examples: brunch (breakfast + lunch), Chimerica (China + America), staycation (stay + vacation), webzine (web + magazine), Internet (international + network), netizen (net + citizen). These new English net words are completely accurate, and they are more in line with the English pronunciation habits. The compound reflects the dual aesthetic views of Chinese and English, so the compound has been liked, used and spread by the on-line people. Meanwhile, the compound words are expanding the English vocabulary too.

\subsection{Old Words with New Meaning}

With the development of network technology, all kinds of new things and new concepts also emerge at the same time, which requires a large number of new words to express. People make use of the existing words and give them a new meaning, and many words are converted from the literal meaning figuratively. Because network English is lively and vivid, such word formation is very common in English net language.

The original meaning of "mouse" is a small furry animal with a long tail, but now a "mouse" is also a device that is connected to a computer. A "window" means an opening in the wall of a building, usually covered with glass. While in computer language, it is usually described as a rectangular part of a computer screen. A "firewall" is designed to prevent the spread of fire through a building or a vehicle, now people use "firewall" to describe a computer system or program. A "bug" originally is the general term for any insect, but it was later extended to a fault or defect in a system or machine.

\subsection{Clever Use of Abbreviations}

In the Internet times, many Internet users give more emphasis on the use of English abbreviations in the network chat in order to save time. This is a popular fashion to achieve more efficient and convenient online communication, The simplified words can be spread to the most degree in the network space, which can not only make the network communication more entertaining, but also transmit the maximum amount of information in the shortest possible time.

Abbreviations mainly include two abbreviation forms: the abbreviation of name and abbreviation of phrase or sentence. There are some examples of name abbreviation: HTTP (Hyper Text Transmission Protocol), BBS(Bulletin Board System), WWW (World Wide Web), TCP(Transmission Control Protocol), PPP (Point to Point Protocol), HTML (Hyper Text Markup Language).

When dealing with the phrase or sentence which has complete meaning, abbreviation is shortening a part of the letters to constitute a new word or only select the first letter of a phrase or a sentence to form new words, such as BTW(by the way), BF(boy friend), FTF(face to face), VG(very good), ATM(automated teller machine), CBD(central business district),GL(good luck), BS(big smile), $\mathrm{AD}$ (advertisement), ASAP(as soon as possible), TCOY(take care of yourself), WTF(what the fuck), OMG(oh my god).

\subsection{Use of Emoticons}

In face to face communication, the receivers receive most of the information through the visual impression including the speaker's gestures or facial expressions, but in the network chat, people 
mostly communicate through the screen. Therefore, the situation that the language fails to express the meaning can hardly be avoided, and sometimes it can cause unnecessary misunderstanding. In order to increase the fun in communication and avoid misunderstandings, users developed a set expression culture.

In today's network communication, emoticons refer to a graphic image which is constituted by numbers, punctuation or symbols of the keyboard. And this word-building mostly use human-like facial expressions to express emotions or feelings simply. To an extent, emoticon can compensate for communication lack of language.“: )” is the smile which stands for smiling face and friendliness, while "ZZZ"stands for feeling boring or sleepy.

Beyond that, the expression package is very popular among the majority of users in recent years. Expression package refers to a variety of small pictures used to transfer the emotional feelings in the process of online chat. And almost all the expression packages are very funny.

\section{Forming Reasons of English Net Language}

\subsection{Popularity of the Internet}

The Internet is the 20th century's greatest inventions of mankind. Its emergence has a huge impact on the world and has greatly changed people's lives. The rapid development of the Internet is an objective condition of forming network English. After decades of rapid development, the Internet has become the computer information network which has the largest and most extensive coverage, the most abundant information resources, the most complete information types, and the most efficient speed of information dissemination. The new English net words emerge in an endless stream, and these words have become essential words when Internet users want to express their thoughts.

As everyone knows that the new discoveries, new inventions and new theories of science and technology all need words to express. Thus, on the one hand, a large number of new words have emerged. Some of them are constructed in combination, while some are transformed basing on the existing words. On the other hand, some of the old words have been given the new meaning which means that the meaning of the word has changed. In this sense, the rapid development of the Internet is the objective condition for the formation of English new words.

\subsection{Increasingly Growing Internet Users}

As a matter of fact, abandoning the old for the new is people's instinct. What causes the evolution of the words is the users always ask for the difference, change and innovate.

According to the data released by the Internet Center: of all the cyber citizens, Internet users whose education degree in high school to college education account for more than 90 percent and most of them are young people. These young people think things actively, and the ability of them to accept new thing is generally strong. For the young creative people, the free and easy environment of network has provided a very good platform. Due to the traditional handwriting is very different from the use of keyboard and text input system, the speed of input was limited. On the other hand, it also formed a alternative writing in network language.

With the advent of the knowledge economy society, the demand of people for knowledge is constantly increasing, and network provides the best way for people to understand the latest information. What's more, the people eager to know what is going on around the world. So there are more and more Internet users, which result in the forming of a large social groups and also giving an important condition to form the Internet English neologisms.

\subsection{Requirement of Fast-paced Life}

With the rapid development of the information age, modern people's pace of life has gradually accelerated. The fast pace of life makes people transmit information as possible as efficiently. So it is necessary to use simple terms to improve input speed, and it is also the inevitable requirement of online communication. Network is a special language carrier which needs to save as much as possible redundant information and to choose the simplest and quickest way to transmit and receive information in the process of delivering verbal information.

In order to transmit the maximum amount of information in the shortest time, there is no doubt that cyber citizen want to find the most efficient expression. To realize the maximum economic 
efficiency, they use the existing figures, letters and symbols on the keyboard to shorten the chat time of network. Generally, as long as the symbols which are knocked out in short time have the function of expression, net citizens think they achieve the purpose of communication.

Concise and arbitrary network English is primarily produced by doing abbreviation or derivation for words or phrases of the English language in accordance with the law of word formation. These words tend to be more innovative, and they are unique, simple and easy to interpret and remember, so the majority of Internet users soon accept and circulate network English in the network communications.

\section{Summary}

Today, the information and knowledge are developing rapidly, and all of the human social activities depend on the network. During the process of communication and exchange, some expressions which are used to show the special meaning do not conform to grammatical rules or expressions.

However, these expressions usually belong to the potential form of English. And I believe they could not only be accepted by public but also become a part of Standard English with the development of English language. At the same time, the development of society will constantly give the vitality to the English network language, and the application of English net language can also increase the expressiveness of language and enrich vocabulary of language.

\section{References}

[1]. Adam, V. An Introduction to Modern English Word-formation. London: Longman, 1973, 89.

[2]. Algeo, John. Fifty Years among the New Words: a Dictionary of Neologism 1941-1991. Cambridge University Press, 1991.

[3]. Crystal, D. Language and the Internet. London: Cambridge University Press, 2006.

[4]. David Crystal. Language and Internet. Cambridge University Press, 2001.

[5]. Halliday, M. Language as Social Semiotic: The Social Interpretation of Language and Meaning. Beijing: Beijing Foreign Language Teaching and Research Press, 2001.

[6]. Jason, H. Lexicography: An Introduction. London/ New York: Routledge, 2002. 